\title{
Treatment of Severe Malaria in the United States
}

\author{
Chandy C. John, MD, MS \\ American Society of Tropical Medicine and Hygiene Washington, DC
}

TO THE EDITOR:

The American Society of Tropical Medicine and Hygiene (ASTMH) shares Krey and Travassos' (1) concerns about treatment of severe malaria in the United States. Since Eli Lilly and Company announced that they would discontinue production of quinidine, the ASTMH has been discussing with the Centers for Disease Control and Prevention (CDC) and U.S. Food and Drug Administration (FDA) how together we can best ensure that patients with severe malaria in the United States have rapid access to highly effective treatment. The CDC and FDA have been working together to address this issue and facilitate the availability of intravenous artesunate to these patients. We anticipate that these organizations will provide additional information on this important issue soon.

Quinine and quinidine are less effective than artesunate for treatment of severe malaria, and continued production of quinidine was not feasible for Eli Lilly and Company. The long-term goal for all groups is for artesunate to be directly available for U.S. hospitals for this purpose, and there has been progress in this area. La Jolla Pharmaceutical has received orphan drug status for this agent (known as LPC-0118) from the FDA and had planned to file a new drug application for it in the fourth quarter of 2019 (2).

In addition, the CDC has published updated guidelines on how to treat severe malaria (3) that address some of the issues raised by Krey and Travassos, including administering oral treatment before artesunate and using a nasogastric tube if oral treatment is not possible. Artesunate therapy can be obtained by calling the CDC Malaria Hotline at 770-488-7788 Monday through Friday between 9:00 a.m. and 5:00 p.m. Eastern time or 770-488-7100 outside of these hours.

We concur that the time required to transport artesunate from the CDC creates an additional hurdle that is concerning. We also strongly support FDA approval of a new formulation of this agent as soon as

This is the author's manuscript of the work published in final edited form as:

John, C. C. (2020). Treatment of Severe Malaria in the United States. Annals of Internal Medicine, 172(3), 224-225. https://doi.org/10.7326/L19-0674 
possible, as we outlined in a letter to the FDA in June 2019. We appreciate both the FDA's efforts to facilitate the availability of intravenous artesunate and the work that the CDC has done to create an interim solution that makes the most effective drug for severe malaria available to patients.

The ASTMH takes clinicians' concerns about treatment of severe malaria in the United States seriously. We are working with our key partners to ensure that patients receive the needed treatment in a timely fashion and that, in the long term, an FDA-approved medication will become available in U.S. hospitals to treat this condition. 


\section{References}

1. Krey RA, Travassos MA. Severe malaria treatment in the United States at the precipice. Ann Intern Med. 2019;171:362-3. [PMID: 31426092] doi:10.7326 /M19-1144

2. Mace KE, Arguin PM, Lucchi NW, et al. Malaria surveillance: United States, 2016. MMWR Surveill Summ. 2019;68:1-35. [PMID: 31099769] doi:10.15585/mmwr.ss6805a1

3. Conn JE, Grillet ME, Correa M et al. Malaria transmission in South America: present status and prospects for elimination. In: Manguin S, Dev V, eds. To-wards Malaria Elimination: A Leap Forward. IntechOpen. 2018. doi:10.5772/intechopen.76964

4. Grillet ME, Hernandez-Villena JV, Llewellyn MS, et al. Venezuela's humanitarian crisis, resurgence of vector-borne diseases, and implications for spillover in the region. Lancet Infect Dis. 2019;19:e149-61. [PMID: 30799251] doi:10 .1016/S1473-3099(18)30757-6

5. Menard D, Dondorp A. Antimalarial drug resistance: a threat to malaria elimination. Cold Spring Harb Perspect Med. 2017;7. [PMID: 28289248] doi: 10.1101/cshperspect.a025619 